\title{
Photoelectron Diffraction for a Look inside Nanostructures
}

\author{
Jürg Osterwalderª, Anna Tamai ${ }^{b}$, Willi Auwärter ${ }^{c}$, Milan P. Allan ${ }^{a}$, and Thomas Greber ${ }^{a}$
}

\begin{abstract}
The diffraction of core-level photoelectrons in local atomic clusters leads to pronounced intensity anisotropies in the corresponding photoemission signals from single crystalline surfaces. The resulting emission patterns contain detailed structural information of the environment of the photoemitting atom. In the case of surface-supported nanostructures, core levels of different elements can be selected in order to probe the local structure around the different constituents. The combination with scanning tunnelling microscopy data, showing the distribution, size and shape of the nano-objects, proves to be very interesting. Three case studies illustrate the kind of information that is available from this combined nano-analysis: molecular orientation in well-ordered chains of $\mathrm{C}_{60}$ molecules on a vicinal $\mathrm{Cu}(111)$ surface, the atomic structure of line defects in epitaxial monolayers of hexagonal boron nitride $(h-\mathrm{BN})$ on $\mathrm{Ni}(111)$, and the structural characterization of two orthogonal one-dimensional $h$-BN and boron phases grown on $\mathrm{Mo}(110)$.
\end{abstract}

Keywords: Boron nitride · Fullerenes · Molecular layers · Nanowires · Self-assembly · Structural defects · Surface analysis · Surface structure

\section{Introduction}

Bottom-up formation by self-assembly processes on surfaces represents an important route for producing arrays of identical nanostructures on a large scale [1]. The ultrahigh-vacuum based instrumentation of surface science offers a rich palette of preparation methods and characterization tools for this purpose. The most widely used analytical methods are scanning tunnelling microscopy (STM) for real space

\footnotetext{
${ }^{*}$ Correspondence: Prof. Dr. J. Osterwaldera

Tel.: +41446355827

Fax: +4144635 5704

E-Mail: osterwal@physik.unizh.ch

aphysik-Institut

Universität Zürich,

Winterthurerstr. 190

$\mathrm{CH}-8057$ Zürich

${ }^{\mathrm{b} C e n t r e}$ for Science

at Extreme Conditions

University of Edinburgh

Mayfield Road

Edinburgh EH9 3JZ, UK

CAMPEL,

Department of Physics and Astronomy

University of British Columbia

2355 East Mall

Vancouver, BC V6T1Z4, Canada
}

imaging on the atomic scale, low-energy electron diffraction (LEED) for measuring surface periodicities, and X-ray photoelectron spectroscopy (XPS) for surface chemical analysis. The latter method can be expanded into a structural probe with chemical selectivity by measuring the angular dependence of core-level intensities. $\mathrm{X}$-ray photoelectron diffraction (XPD) effects lead to strong intensity anisotropies that reflect the site geometry around the photoemitting atoms [2]. These scattering and interference processes are effective only within a relatively short range of the order of 1 to $2 \mathrm{~nm}$ due to the strong inelastic attenuation of the photoelectron waves. In order to produce measurable anisotropies for a macroscopic sampling area (typically one to a few $\mathrm{mm}$ ), no long range crystallographic order is thus required, but long range orientational order of small, crystallographically identical units. The technique is therefore ideally suited to look into the atomic structure of nano-objects that are dispersed on a surface with orientations fixed by the interaction with a crystalline substrate. Typical situations of this kind include molecular layers [3], defect structures in ultrathin films [4] and surface superstructures with large unit cells [5]. In this paper, a few case studies illustrate the detailed structural information that can be gained by combining STM and XPD measurements. While STM provides the distribution, size and shape of the objects, the anisotropies in
XPD data are generated inside the objects and yield information about their crystallographic structure.

\section{A Photoelectron Diffraction Primer}

The principles of XPD have recently been reviewed [6], therefore we give only a brief account here. Experimentally, a commercial XPS spectrometer is used in order to record photoemission core-level spectra, with the prerequisite that the angular acceptance cone for photoelectrons can be restricted to half angles of the order of $1^{\circ}$ to $3^{\circ}$. In the typical XPD experiment, the electron analyzer is mounted in a fixed geometry on the ultrahigh-vacuum chamber of the spectrometer, while the single crystalline samples are sitting on a computer-controlled goniometer [7]. In an automated fashion, the intensities of selected core-level peaks are recorded while the goniometer moves the sample either along a polar or an azimuthal angular scan, or both, relative to the analyzer acceptance direction. When both angles are scanned in a sequential way, the full hemispherical diffraction pattern for the particular core levels is obtained [8], such as those presented in the following sections. Depending on the signal intensity and the degree of the angular anisotropies, full scans take from 30 min up to $24 \mathrm{~h}$ or more, such that sample stability may be a concern. 
XPD data can be modelled by scattering calculations, considering a coherent superposition of the primary excited photoelectron wave originating from a particular atom with the singly and multiply scattered waves emanating from all atoms within a limited cluster surrounding the photoemitter [9]. The cluster approach is fully adequate due to the strong inelastic attenuation of the photoelectron waves, and it has been very successful in describing XPD diffraction patterns of well-defined and known structures [10]. In a typical structure search, the cluster geometry is varied in a systematic or intuitive way until the calculated pattern best matches the experimental one, where 'best match' is usually quantified by a suitable reliability factor [10].

XPD patterns often contain characteristic fingerprints that convey a qualitative (or semi-quantitative) starting point for a structural model [6]. The most prominent fingerprint arises from the highly anisotropic form factor for scattering of electron waves off a single atom: at the typical photoelectron energies in the range from 300 to $1500 \mathrm{eV}$, it is strongly peaked in the forward direction. Therefore, interatomic vectors pointing from the photoemitter to neighbouring atoms are reflected by pronounced intensity maxima, seen as bright spots in the stereographically projected grey-scale plots of the patterns. From these forward-scattering peaks, bond directions in adsorbed molecules [11] or in ultrathin films [12] become directly visible in the raw data. In favourable cases, information on bond lengths is available from the observation of circular fringes that arise due to the axial symmetry of individual emitterscatterer pairs and the constructive interference between primary and singly-scattered photoelectron waves. They are centered at forward-scattering maxima and are visible most clearly in XPD patterns from single monolayer adsorbates where they are not overwhelmed by other forward-scattering maxima [13]. For adsorbate atoms sitting on a heavy, strongly scattering substrate, the enhanced backscattering amplitude can be exploited to observe the higher-order fringes for photoelectrons emitted from an adsorbate atom and backscattered by a substrate atom just underneath [14]. From these, very precise absorbate-substrate bond-length information can be extracted. However, this requires measurements at low temperatures in order to minimize the Debye-Waller effect that is very strong in backscattering.

\section{A Look into a Molecular Layer}

Well-ordered molecular layers on patterned surfaces represent an important class of nanostructures, where specific supramo- lecular structures can be induced by the underlying template [5][15]. The properties of such layers depend not only on the two-dimensional molecular arrangement, but also on the orientation of the individual molecules and on conformational changes induced by the adsorption. The combination of STM and XPD is rather powerful for providing a complete characterization of such systems. For illustration, we discuss the case of fullerene chains formed on a stepped copper surface.

$\mathrm{Cu}(553)$ is a surface vicinal to $\mathrm{Cu}(111)$ which exhibits $9.8 \AA$ wide terraces of (111) orientation separated by equidistant monoatomic steps (see Fig. 1d). Such one-dimensional template surfaces are easily prepared by cutting a crystal surface at a small miscut angle relative to the low-index (111) planes (here it is $12.3^{\circ}$ ). A molecular monolayer is prepared by evaporating $\mathrm{C}_{60}$ onto this template and subsequent annealing at $570 \mathrm{~K}$ for a few minutes [15]. The terraces are just wide enough to accommodate one row of $\mathrm{C}_{60}$ molecules, and the formation of chains along the steps is therefore no surprise. However, STM images display a structure with two alternating inequivalent chains (Fig. 1a), which are imaged with different brightness. XPD has been instrumental in solving the puzzle of the internal chain structure leading to this modulation of chain topography. The $\mathrm{C} 1 \mathrm{~s}$ diffraction pattern is given in Fig. 1b. At close inspection it displays both three-fold and five-fold elements with respect to the terrace normal (indicated by the small circle near the centre of the pattern), hinting at a coexistence of two inequivalent molecular orientations.

For the further discussion of this pattern, one should note that the diffraction pattern from an individual $\mathrm{C}_{60}$ molecule is largely dominated by the intramolecular forward scattering within a single $\mathrm{C}_{60}$ cage [3]. It is thus directly reflecting the orientation of the molecule. The situation is similar, but not equivalent, to placing a projector lamp at the centre of a model molecule and observing the shadow pattern produced on a large screen. In fact, we have 60 lamps at the atom positions forming the cage and observe the complex shadow pattern that still retains the symmetry of the molecule. And, to put this simple analogy into its proper context, forward scattering enhances the photoelectron flux along an interatomic direction rather than producing a shadow [3].

The $\mathrm{C}_{60}$ cage is rather stiff, and we do not expect significant distortions in the molecular geometry upon adsorption. Moreover, intermolecular scattering between different molecules is weak due to the thermal relative motion and the long scattering distance, and backscattering of photoelectrons from the underlying substrate is weak due to the large scattering angles. The three Eulerian angles that define the molecular orientation are then the only degrees of freedom that are relevant to describe the XPD pattern from $\mathrm{C}_{60}$ layers. In the current situation, where coexisting orientations are suspected, the patterns associated with the different orientations can simply be superposed, with weight factors according to the relative abundance of the different 'species'. Fig. 1c illustrates the success of this procedure: the pattern obtained by SSC calculations for a 50:50 percent mixture of molecules bonded to the (111) terraces by pentagon bonds or by hexagon bonds reproduces all the salient features of the experimental data of Fig. 1b [16]. The structural model given in Fig. 1d is then obtained in a straightforward manner. Although the XPD results do not tell us the spatial distribution of hexagon- and pentagon-bonded molecules, the alternating chain images observed in the STM make the link between chain topography and molecular orientation rather obvious.

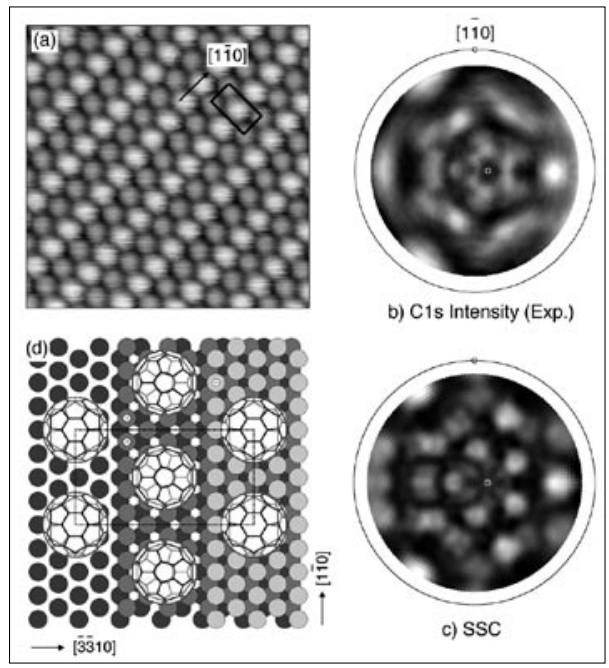

Fig. 1. a) Room temperature STM image (100 $\AA$ $\times 100 \AA$ ) of $C_{60}$ chains forming on $\mathrm{Cu}(553)$. b) Experimental C1s XPD pattern (Mg K $\alpha, \mathrm{E}_{\mathrm{kin}}=970$ $\mathrm{eV})$. The chain direction and the direction normal to the terraces are indicated. c) SSC calculations for a one-to-one mixture of pentagon-bonded and hexagon-bonded $\mathrm{C}_{60}$ molecules. d) Realspace model of the chain geometry suggested by these results. The rectangular surface unit cell is marked in parts a) and d) (Figure taken from A. Tamai, A.P. Seitsonen, T. Gerber, J. Osterwalder, Phys. Rev. B 2006 74, 085407. Copyright (2006) by the American Physical Society).

Another subtlety in this model is that, for the hexagon-bonded molecules, there are actually two inequivalent orientations that should be degenerate in adsorption energy. When they are rotated by $180^{\circ}$ about the three-fold molecular axis, the bottom hexagon can bond in a very similar fashion to the terrace. This degeneracy had to be considered in producing the theoretical pattern by actually using $a h_{1}: h_{2}: p=25: 25: 50$ mixture of the two hexagon-bonded orientations $\left(\mathrm{h}_{1}, \mathrm{~h}_{2}\right)$ and of the pentagon-bonded 
one (p) [16]. In recent low-temperature STM experiments [17] this model is nicely confirmed. Intramolecular structures are imaged and show the presence of three different orientations. One chain consists of molecules with a single orientation, while the adjacent chains display molecules with two different orientations, without obvious ordering.

The structural peculiarities of this dualchain system are reflected in the interesting electronic properties. The unusual pentagon-bonded chains exhibit strongly one-dimensional character and a large electronic band width along the chains [16]. Electron hopping is enhanced by the strong wavefunction overlap of molecular orbitals along the chains, favoured by the particular orientation of the individual molecules and the high structural coherence.

\section{A Look at Defects in a Single- Layer Insulator}

In silicon-based microelectronics, the characterization and control of interfaces was a key step for the development of electronic devices over the last four decades. In nanostructures, most atoms are part of or very near to an interface between two different materials. The atomic structure of such interfaces is determinant for their behaviour in a nanoscopic device as it influences the electrical transport or magnetic coupling across the interface. The combination of XPD and STM provides a powerful tool for characterizing interfaces on the atomic scale.

Monolayer films of hexagonal boron nitride $(h-\mathrm{BN})$ on transition metal surfaces represent an interesting case of an ultimately thin metal-insulator interface. Such films can be grown in great perfection by thermal decomposition of borazine $(\mathrm{HBNH})_{3}$ precursor molecules on the clean and hot (typically $1000 \mathrm{~K}$ ) transition metal surface [18]. The process depends on the catalytic activity of the transition metal and is thus selfterminating at one monolayer (ML). The layer exhibits a large band gap comparable to the $5.2 \mathrm{eV}$ in bulk $h$-BN. The strong $\mathrm{sp}^{2}$ intralayer bonds make the layer very stiff and lead, in cases of large lattice mismatch, to the formation of complex and highly regular nanostructures. The most notable example is the boron nitride nanomesh that forms on $\mathrm{Rh}(111)$ [5].

On $\mathrm{Ni}(111)$ there is a good lattice match, and upon saturation the $h$-BN layer covers uniformly the entire surface, with atomically flat terraces of up to several hundred nanometres in size. The structure is a $(1 \times 1)$ $h$-BN honeycomb, with one $\mathrm{N}$ atom on top of every surface Ni atom (Fig. 2c), and with $\mathrm{B}$ atoms situated in the so-called fcc sites of the $\mathrm{Ni}(111)$ surface, in a plane slightly $(-0.1 \AA)$ below the $\mathrm{N}$ atoms [19-21]. This corrugation in the $h$-BN layer is directly observed in B 1s XPD patterns, where it gives rise to three intensity maxima along the B-N nearest-neighbour bonds at shallow emission angles ([20], see also Fig. 2a). In a recent density functional theory (DFT) calculation, this structure was found to have the lowest total energy, but it was pointed out that a similar structure, with B atoms in hcp sites, has a total energy that is only marginally ( $9 \mathrm{meV}$ per $\mathrm{BN}$ unit) higher [22]. In a later study Auwärter et al. [4] showed that it is possible to prepare submonolayer $h$-BN coverages on $\mathrm{Ni}(111)$ that display predominantly one of these two phases. In terms of the $h$-BN film alone, the two domains are related to each other by a simple azimuthal rotation by $180^{\circ}$ ( $c f$. Figs. 2c and f). Accordingly, the three intensity maxima in the $B$ 1s XPD pattern appear rotated by $180^{\circ}$ (Fig. 2d) [23]. The different crystallographic structures are also reflected in the overall island shapes, as is shown by the STM conductance images in Figs. $2 \mathrm{~b}$ and e. In this imaging mode, the lower conductance through the $h$-BN clearly identifies the boron nitride islands, which appear with triangular shapes of up to $200 \mathrm{~nm}$ in diameter. The two preparations lead to triangles that are rotated by $180^{\circ}$ relative to each other. From knowing the absolute orientation of the sample in both XPD and STM studies, the island models of Fig. 2c and $f$ can be plotted, indicating that one particular type of island edge is stable. It is assumed that the $\mathrm{N}$ termination is the stable one, because it produces fewer broken bonds.

Upon close inspection of STM images of full-monolayer $h$-BN films, a low concentration of line defects has been observed. These line defects are found to play an important role in metal deposition and cluster aggregation on top of such films. Small three-dimensional clusters form upon room temperature deposition of Co and align themselves along these line defects [24], while for deposition at elevated temperatures $(450 \mathrm{~K})$ these defects act as entry sites for Co intercalation underneath the $h$-BN layer. They represent thus active sites for the nano-engineering of metal-insulator-metal structures. Preparations of submonolayer $h$-BN films under conditions that produce both types of $h$-BN structures in coexistence helped to understand the detailed structure of these line defects. As shown in Fig. 3b, the defects appear just where two triangular islands of different domains touch one another. From our knowledge of the island termination (Fig. 2) a detailed structural model for the line defect can be deduced (Fig. 3c). In this atomic model it is recognized that the nitrogen sublattice is continuous across the defect line, while the boron sublattice exhibits an antiphase domain boundary. By establishing the defect structure down to the atomic level, the combination of STM and XPD data provides a basis for the understanding of processes like the anchoring of nanoclus-

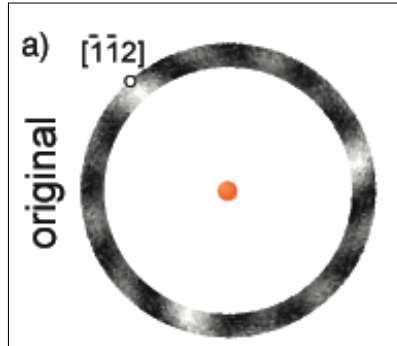

B 1s XPD

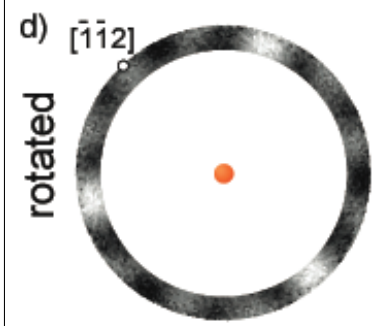

b)

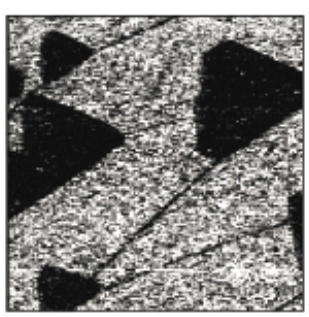

conductivity

e)

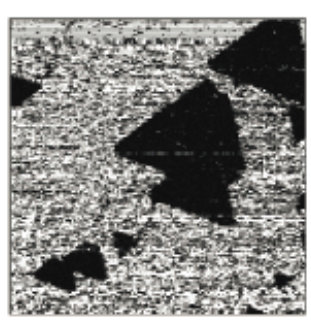

c)

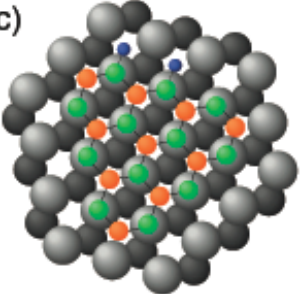

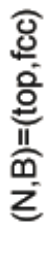

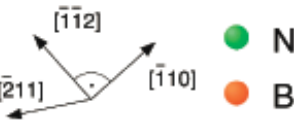

f)
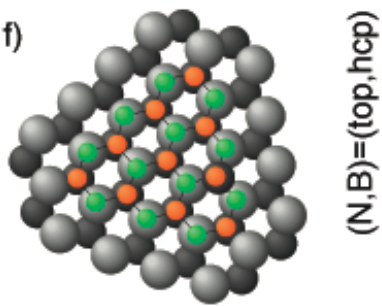

Fig. 2. XPD and STM data from submonolayer $h$-BN films exhibiting triangular islands; a) and d) represent Si K $\alpha$ excited B 1s XPD patterns for polar angles $\theta$ from $86^{\circ}$ to $78^{\circ}$ and for two different film preparations resulting in predominantly one or the other type of two crystallographic $h$-BN domains (see c) and f) for illustrations of the two structures); b) and e) give STM conductance images (400 $\mathrm{nm} \times$ $400 \mathrm{~nm}, \mathrm{~V}_{\mathrm{B}}=-0.6 \mathrm{~V}, \mathrm{I}_{\mathrm{T}}=3 \mathrm{nA}$ ) for the same preparations. In the structural models of c) and f), first-layer (bright gray spheres) and second-layer (smaller, dark gray spheres) $\mathrm{Ni}$ atoms represent the $\mathrm{Ni}(111)$ surface. In c) two hydrogen atoms (small blue spheres) indicate a possible hydrogen decoration of the island edges (from Ref. [4]). 


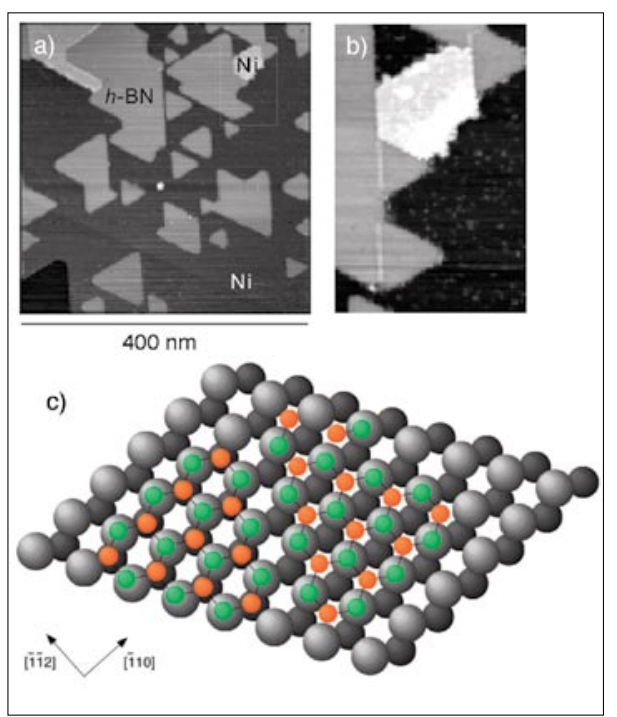

Fig. 3. a) STM image $\left(310 \mathrm{~nm} \times 310 \mathrm{~nm}, \mathrm{~V}_{\mathrm{B}}=-0.2\right.$ $\mathrm{V}, \mathrm{I}_{\mathrm{T}}=1 \mathrm{nA}$ ) for a submonolayer $h$-BN coverage on $\mathrm{Ni}(111)$ prepared under conditions such as to produce both types of islands discussed in the text. b) Enlarged image of the area marked in a), using the same tunnelling conditions. The bright patch in the upper half of the image represents a second $\mathrm{Ni}$ layer. c) Atomic model of the defect forming along the line where two different domains meet (from Ref. [4]).

ters and the intercalation of metal atoms underneath the $h$-BN film.

\section{A Look into One-Dimensional Boron Nitride and Boron Nanostructures}

New nanostructured phases are formed upon high-temperature decomposition of borazine on transition metal surfaces. Among other factors, it is the lateral stiffness of the $\mathrm{sp}^{2}$-bonded, graphene-like $h$ $\mathrm{BN}$ sheets in concert with a large lattice mismatch, or a symmetry-mismatch on a non-hexagonal substrate, that drives the break-up of the layer, often under formation of regular nanostructures [5]. The (110) surface of molybdenum represents an interesting case because the surface layer of this body-centered cubic metal is almost but not quite hexagonal: it results from a uniaxial compression of a hexagonal layer by $18.4 \%$ along the $\left[\begin{array}{lll}1 & -1 & 0\end{array}\right]$ direction. This anisotropic deviation from hexagonal symmetry has raised expectations for the formation of a one-dimensional (1D) superstructure.

Experimentally, it is found that borazine decomposition on $\operatorname{Mo}(110)$ leads to the formation of two different $1 \mathrm{D}$ nanostructures, depending on the surface temperature [25]. On samples prepared at $950 \mathrm{~K}$, STM images display dense arrays of narrow stripes running along the $[1-1$ 0] direction (Fig. 4a). From XPS and angle-resolved photoemission spectroscopy (ARPES) measurements the presence of a
$h$-BN layer can be concluded, and LEED patterns show a $(4 \times 1)$ periodicity along the [001] direction, i.e. the layer is commensurate with the molybdenum surface. The B 1s XPD pattern from this stripe phase (Fig. 5a) shows the typical characteristics seen also in other $h$-BN monolayer systems: the intensity maxima along the B-N nearest-neighbour bonds at shallow emission angles (see also Figs $2 \mathrm{a}$ and d), and no significant intensity anisotropies near the centre of the plot [20]. The absence of forward scattering maxima for lower polar angles indicates that the boron nitride is present as a single layer. Four dominant intensity maxima spaced by $60^{\circ}$ and $120^{\circ}$ in azimuth appear at a polar angle of $78^{\circ}$. Actually, two equivalent maxima

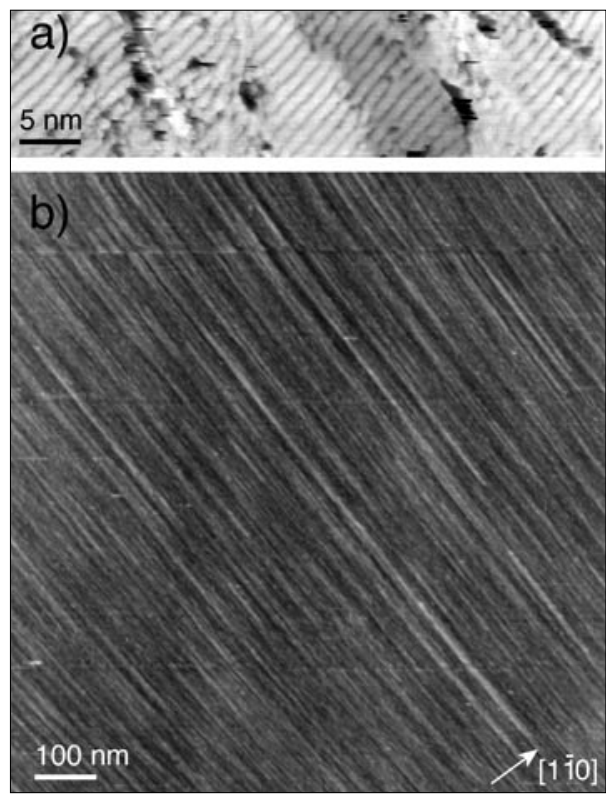

Fig. 4. a) STM image of $h$-BN stripes prepared by borazine decomposition on $\mathrm{Mo}(110)$ at $950 \mathrm{~K}$ $\left(V_{B}=-3.0 \mathrm{~V}, I_{T}=0.6 n A\right)$. b) STM image of boron nanowires prepared by borazine decomposition on $\mathrm{Mo}(110)$ at $1112 \mathrm{~K}\left(\mathrm{~V}_{\mathrm{B}}=1.0 \mathrm{~V}, \mathrm{I}_{\mathrm{T}}=1.0 \mathrm{nA}\right.$; Figures taken from Ref. [25]).

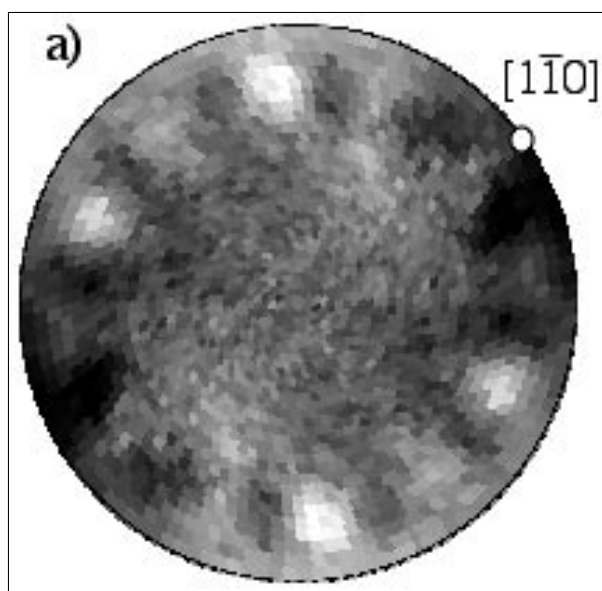

are likely to be present also along the [ $1-10]$ axis, but they are partly shadowed by two mounting screws next to the sample. The polar angle is lower than for $h$ $\mathrm{BN}$ on $\mathrm{Ni}(111)$ where they are centered at $86^{\circ}$ [20], suggesting that the layer is more strongly corrugated on $\mathrm{Mo}(110)$. The pattern is here also more diffuse which is in line with the much higher defect density observed in the STM images.

At higher preparation temperatures, the borazine decomposition proceeds further and the nitrogen disappears from the surface. After preparation at $1112 \mathrm{~K}$, XPS spectra show only boron and molybdenum signals, and STM images display the result of an astounding restructuring of the surface in the form of extremely long and thin straight wires. They run along the [001] direction of the substrate, i.e. in the direction perpendicular to the stripes of the $h$-BN phase, and are between 2 and 10 $\mathrm{nm}$ wide and often more than $1 \mu \mathrm{m}$ long. This boron nanowire (BNW) phase exhibits perfect atomic order along the wires but no order perpendicular to them, as is evidenced by the LEED patterns. The B 1s XPD pattern of Fig. 5b illustrates the different local structure around the boron atoms in this phase. Two forward scattering maxima at grazing emission can be associated with neighbouring atoms along the wires, and the appearance of rather sharp circular fringes centered at these maxima is a further indication for the high degree of atomic order in this direction. More diffuse maxima are spread over the full emission hemisphere and indicate that there is also a substantial amount of boron situated on octahedral sites of the Mo lattice in the surface region [25]. Although a more quantitative structural model for the BNW phase has not yet resulted from these data, they nevertheless provide important information about the structural elements within this remarkable nanostructure.

Fig. 5. B 1s XPD patterns measured on a Mo(110) surface covered by $h$-BN stripes a) and B nanowires b) as shown in Fig. 4. The patterns are oriented in the same way as the data of Fig. 4 (taken from Ref. [26]). 


\section{Outlook}

The presented case studies illustrate the potential for the combined use of STM and XPD in performing detailed materials science studies in nanostructures and on the single monolayer level. The specificity of XPD to specific regions in these structures can be further enhanced by exploiting the chemical shifts in core-level spectra. XPD patterns can thus be measured for photoemitters of one element in different chemical environments. This has been applied, e.g. to characterise a mixture of $h-\mathrm{BN}$ and BNW phases on $\mathrm{Mo}(110)$ prepared at intermediate temperatures [26].

The use of XPD is much less widely spread in the surface science community than STM, probably because the technical upgrades needed to turn an XPS spectrometer into an XPD diffractometer are not commercially available, and because the data analysis is often more cumbersome. However, there are several user facilities at synchrotron radiation centres, such as the Near-Node Photoelectron Holography endstation at the Surface and Interface Microscopy (SIM) beamline of the Swiss Light Source, that provide general access to excellent XPD instrumentation on a scientific proposal basis. This instrument has demonstrated its sensitivity to detailed and non-trivial adsorption geometries of small molecules [27].

\section{Acknowledgements}

This work has been supported by the Swiss National Science Foundation and by the European Union's Sixth Framework Programme via the NanoMesh project (NMP4-CT-2004013817)

Received: September 4, 2006

[1] J.V. Barth, G. Costantini, K. Kern, Nature 2005, 437, 671-679.

[2] L.-G. Petersson, S. Kono, N.F.T. Hall, C.S. Fadley, J.B. Pendry, Phys. Rev. Lett. 1979, 42, 1545-1548.

[3] R. Fasel, P. Aebi, R.G. Agostino, D. Naumovic, J. Osterwalder, A. Santaniello, L. Schlapbach, Phys. Rev. Lett. 1996, 76, 4733-4736.

[4] W. Auwärter, M. Muntwiler, J. Osterwalder, T. Greber, Surf. Sci. Lett. 2003, 545, L735-L740.

[5] M. Corso, W. Auwärter, M. Muntwiler, A. Tamai, T. Greber, J. Osterwalder, Science 2004, 303, 217-220.

[6] J. Osterwalder, in 'Surface Analysis by Auger and X-Ray Photoelectron Spectroscopy', Eds. D. Briggs, J.T. Grant, IM Publications and SurfaceSpectra Limited, Chichester, 2003, p. 557-585.
[7] T. Greber, O. Raetzo, T. J. Kreutz, P. Schwaller, W. Deichmann, E. Wetli, J. Osterwalder, Rev. Sci. Instrum. 1997, 68, 4549-4554.

[8] J. Osterwalder, T. Greber, A. Stuck, L. Schlapbach, Phys. Rev. B 1991, 44 , 13764-13767.

[9] A.P. Kaduwela, D.J. Friedman, C.S. Fadley, J. Electron Spectrosc. Relat. Phenom. 1991, 57, 223-278.

[10] R. Fasel, P. Aebi, L. Schlapbach, J. Osterwalder, Phys. Rev. B 1995, 52, R2313R2316.

[11] R.S. Saiki, G.S. Herman, M. Yamada, J. Osterwalder, C.S. Fadley, Phys. Rev. Lett. 1989, 63, 283-286.

[12] W.F. Egelhoff, Jr., Phys. Rev. Lett. 1987, 59, 559-562.

[13] R. Fasel, P. Aebi, J. Osterwalder, L. Schlapbach, Surf. Sci. 1995, 331-333, 80-87.

[14] T. Greber, J. Wider, E. Wetli, J. Osterwalder, Phys. Rev. Lett. 1998, 81, 1654 1657.

[15] A. Tamai, W. Auwärter, C. Cepek, F. Baumberger, T. Greber, J. Osterwalder, Surf. Sci. 2004, 566-568, 633-637.

[16] A. Tamai, A.P. Seitsonen, T. Greber, J. Osterwalder, Phys. Rev. B 2006, 74, 085407, $1-5$.

[17] A. Tamai, P. Ruffieux, unpublished.

[18] A. Nagashima, N. Tejima, Y. Gamou, T. Kawai, C. Oshima, Phys. Rev. B 1995, 51, 4606-4613.

[19] Y. Gamou, M. Terai, A. Nagashima, C. Oshima, Sci. Rep. RITU A 1997, 44, 211 214.

[20] W. Auwärter, T.J. Kreutz, T. Greber, J. Osterwalder, Surf. Sci. 1999, 429, 229-236.

[21] M. Muntwiler, W. Auwärter, F. Baumberger, M. Hoesch, T. Greber, J. Osterwalder, Surf. Sci. 2001, 472, 125-132.

[22] G.B. Grad, P. Blaha, K. Schwarz, W. Auwärter, T. Greber, Phys. Rev. B 2003, 68, 085404, 1-7.

[23] Up to this point it has not been possible to identify the exact preparation routes to the two different structures. No systematic studies have been done. However, lower carbon contamination of the substrate appears to favour the formation of the B-fcc phase of Figs. 2a-c.

[24] W. Auwärter, M. Muntwiler, T. Greber, J. Osterwalder, Surf. Sci. 2002, 511, 379386.

[25] M. Allan, S. Berner, M. Corso, T. Greber, J. Osterwalder, submitted.

[26] M. Allan, Diploma Thesis, ETH Zürich, 2006.

[27] T. Greber, Z. Sljivancanin, R. Schillinger, J. Wider, B. Hammer, Phys. Rev. Lett. 2006, 96, 056103-1-4. 